\title{
Evidence-based Dentistry International Collaborative Group Derek Richards
}

$\triangle$ $\mathrm{t}$ the recent International Association for Dental Research (IADR) meeting in Vancouver (March 10-14th 1999) there were four very well attended sessions which examined methods for implementing evidencebased dentistry. There was a symposium on evidence-based medicine and dentistry in dental education supported by the International Federation of Dental Education Associations (IFDEA) and American Association of Dental Schools (AADS). The first meeting was held of an international collaborative group on evidence-based dentistry. This was supported by the Agency for Health Care and Policy Research (AHCPR) and the National Institute for Dental and Craniofacial Research (NIDCR).

There was an early morning plenary session on the evidence-based medicine approach and a lunch time symposium from the Cochrane Collaboration on how to conduct evidence-based dentistry. The AADS meeting also had a whole day workshop sponsored by Delta Dental Plan of Massachusetts on an introduction to evidence-based skills development. I believe this heralds the integration of evidence-based health care in dentistry. The meeting also highlighted the fact that we have significant work to do with more than twenty years lag behind medicine.

For example, one paper ${ }^{1}$ indicated that in terms of numbers of randomisedcontrolled trials (RCT's) and metaanalyses conducted, dentistry is a poor performer when compared with most of the medical specialties. One participant suggested anecdotally that only $8 \%$ of dental treatments were based on RCT's. This can be compared to medicine and psychiatry where research indicates that $82 \%{ }^{2}$ and $65 \%{ }^{3}$ of treatments respectively are based on RCT's.
The IADR also saw the initial presentation of findings from a number of systematic reviews on topics such as enamel erosion, oral care for cancer patients, single tooth implants and pre-formed crowns. The largest and most notable is a systematic review completed by a group of researchers from Cardiff on the longevity of dental restorations. ${ }^{4}$ (A summary of this is now available on the $w_{e} b^{5}$ ). In addition to producing clear recommendations some of these reviews are also raising issues relating to the quality of research. These are not merely related to the conduct, but also the planning, analyses, outcome measures and reporting of research. The recent announcement in the BDJ of their adoption of the CONSORT statement ${ }^{6}$ for reporting trials can only help in the development of this.

There were a number of interesting presentations at the first meeting of an international group of dentists interested in promoting dialogue among researchers and educators working in the field of evidence-based dentistry. Nigel Pitts from Dundee, who was awarded the H. Trendley Dean Memorial Award at the IADR meeting, gave an overview of the current activities in the UK. I described the genesis and the activities of the Centre for Evidence-based Dentistry. ${ }^{7}$ The international activities of the Cochrane Oral Health Group currently based in Manchester were outlined by Helen Worthington who is one of the co-ordinating editors of the group. Amid Ismail, who with Jan Clarkson organised this first meeting, discussed progress to date in the USA. Jim Leake, from the host country Canada, gave a very interesting talk on the activities of his unit. There were also many contributions from other countries represented showing that a wide range of initiatives is currently underway. Dushanka Kleinman, the Deputy Director of the NIDCR, was able to announce some further initiatives in the USA for the future. The first of these was a communication strategy which should also help develop and promote the widespread application of evidencebased methods in dental research, education and practice. This supplement will endeavour to play a part in fulfilling this aim.

Much of the early activity in evidencebased dentistry has centred in the UK, stimulated by the formation of the Centres for Evidence-based Medicine and Dentistry that are based in Oxford. The UK also hosts the Cochrane Oral Health Group, an international group engaged in conducting systematic reviews in dentistry, whose activities are described in an article on page 22-23 of this supplement. In addition to this there are a number of other groups of dentists in the UK involved with evidence-based research. A symposium on evidence-based dental activity in the UK is planned in November of this year. ${ }^{8}$ Details will appear on the Centre for Evidence-based Dentistry website shortly. ${ }^{9}$ One of the aims of this symposium will be to assess the wide range of evidence-based activities currently underway in the UK and help form collaborations and co-ordinate activity in order to avoid waste and duplication.

To continue to improve oral health care, work will be required to categorise, synthesise and summarise previously published and newly reported clinical information. This cannot be done in isolation. Evidence-based clinical care must harness the willingness of individuals, research groups and institutions to facilitate this process in a co-ordinated manner both nationally and internationally. These early fledgling initiatives are 
welcome, but collaborations need to deepen and grow to produce results that all health care professionals can use to the benefit of patients.

1 Badovinac R, Conway S, Neiderman R. Bibliometric Analysis of Evidence-based Dental Theraputics literature. IADR Abstract 148.

2 Ellis \}, Mulligan I, Rowe J, Sackett D L. Inpatient general medicine is evidence based. Lancet; 346: 407-10, 1995.

3 Geddes I R, Game D, Jenkins N E, Peterson L A, Pottinger $G$ R, Sackett $D$ L. What proportion of primary psychiatric interventions are based on evidence from randomised controlled trials? Qual in Health Care,1996; 5:

215-217

4 Chadwick B, Dummer P, Dunstan F, et al. A systematic review of the longevity of dental restorations. York: NHS Centre for Reviews and Dissemination, University of York, forthcoming.

5 http://www.york.ac.uk/inst/crd/ehc52.htm

6 http://www.ihs.ox.ac.uk/cebd/

7 Begg C, Cho M, Eastwood S, et al. Improving the quality of reporting of randomised controlled trials. JAMA, 1996; 276: 637-639.

8 CEBD@bhadentph.demon.co.uk

9 http://www.ihs.ox.ac.uk/cebd

\section{Jan Clarkson, Helen Worthington and lain Chalmers} Reducing harm and maximizing benefit

W e would all like to believe that what we do for our patients is for their own good and in their best interests. However, most clinicians can recall instances when a clinical intervention has been more detrimental than beneficial to a patient, and several procedures in dentistry are now considered to have done more harm than good. For example it was once believed that a certain width of attached mucosa was essential for periodontal health. Based on this premise patients were subjected to uncomfortable surgery which exposed bone and resulted in loss of periodontal support. A recent metaanalysis compared surgical versus nonsurgical methods of treating periodontal disease and found that only the deepest pockets benefited from the surgery ${ }^{1}$. The extraction of asymptomatic third molars on a massive scale also seems to have been a waste of resources for patients and the NHS, particularly because it has led to patient morbidity and mortality ${ }^{2}$. Currently, there are questions about the effects of routine scale and polish ${ }^{3}$, the relative merits of different restorative materials ${ }^{4}$ and orthodontic methods ${ }^{5}$.

One problem facing clinicians and patients who want to reduce harm and maximise benefit from dental care is finding relevant, reliable research evidence. It is increasingly difficult for clinicians to locate and assimilate information from the large volume of scientific papers published in peer reviewed journals, not to mention the continuing flow of unsolicited information extolling the virtues of the latest products. Faced with these problems, practitioners have turned to review articles as the most accessible sources of practical advice.

Unfortunately, review articles are often based on an unsystematic search for and evaluation of the relevant research evidence and thus often lead to biased conclusions. The information accessible to patients is perhaps even more biased and usually comes from sensational press coverage of new developments in the dental field. Many clinicians will have experienced a patient arriving for an appointment with a press clipping, asking for information and sometimes requesting a particular treatment.

In the future, it seems likely that consumers who have heard about evidencebased clinical practice will increasingly demand relevant and reliable information before deciding on their treatment options. For both clinicians and patients, the Internet is rapidly becoming a major source of up-to-date information on the effectiveness of clinical procedures. In addition, clinicians may become more actively involved in generating relevant evidence by conducting their own research. The recent push for 